J. Med. Microbiol. - Vol. 25 (1988), 183-186

(C) 1988 The Pathological Society of Great Britain and Ireland

\title{
Slight hyperinsulinaemia but no hypoglycaemia in pertussis patients
}

\author{
B. L. FURMAN, E. WALKER*, FIONA M. SIDEY and A.C. WARDLAW†
}

Department of Physiology and Pharmacology, University of Strathclyde, Glasgow G1 1XW. *Communicable Diseases (Scotland) Unit, Ruchill Hospital, Glasgow G20 9NB, and †Department of Microbiology, University of Glasgow, Alexander Stone Building, Garscube Estate, Bearsden, Glasgow G61 10H

\begin{abstract}
Summary. Because of the central rôle postulated for Pertussis Toxin in the pathogenesis of whooping cough, and the well-established ability of this toxin to alter insulin and glucose levels in animal blood, a study of insulin and glucose levels in hospitalised pertussis patients and in controls was made. With blood specimens collected in heparin-fluoride anticoagulant, the geometric mean plasma-insulin level $(13.3$ $\mu \mathrm{U} / \mathrm{ml}$ ) in a series of 24 pertussis patients was slightly, but statistically significantly, higher than that $(8.9 \mu \mathrm{U} / \mathrm{ml})$ in a series of 27 non-pertussis controls with other infectious diseases $(\mathrm{p}<0.02)$. Portions of the same blood specimens collected in lithium-heparin anticoagulant yielded higher mean plasma-insulin values of 15.5 and $11.4 \mu \mathrm{U} / \mathrm{ml}$ respectively, with no significant difference between them $(\mathrm{p}>0.05)$. Mean plasma glucose concentrations were not significantly different between the two groups, and hypoglycaemia was not detected in any pertussis patient. There were no statistically significant differences between pertussis and control children in the mean levels of plasma calcium, magnesium or phosphate.
\end{abstract}

\section{Introduction}

According to Pittman $(1979,1984)$, pertussis is a toxin-mediated disease in which the cardinal signs are produced by the component of Bordetella pertussis that is now known as Pertussis Toxin (PT) and which has been well-characterised biochemically (Ui et al., 1985). Other names for PT are Histamine-Sensitising Factor (HSF), Leukocytosisand Lymphocytosis-Promoting Factor (LPF), Islets-Activating Protein (IAP) and Pertussigen, each term reflecting particular biological activities of the toxin in experimental animals or in man (Wardlaw and Parton, 1982). For example, the IAP activity is responsible for hyperinsulinaemia in experimental animals (Ui et al., 1985).

In man, the role of PT in the pathophysiology of the disease is not fully established. Nevertheless there is little doubt that, during an infection with $B$. pertussis, PT gains access to the circulation of the patient, because lymphocytosis (absolute or relative) is a well-recognised diagnostic sign (Walker et al., 1981) in the disease and can reasonably be attributed to this toxin. Regarding HSF activity, there is a report (Sanyal, 1960), but also a denial (Pieroni et al., 1971), of histaminesensitisation in children with pertussis.

Received 13 Mar. 1987; accepted 27 May 1987.
From the IAP activity of PT, it might be expected that plasma insulin would be elevated in pertussis; but no supporting data have yet been published. There is, however, the long-standing report (Regan and Tolstoouhov, 1936) of hypoglycaemia in children with pertussis - an effect which could have arisen from excessive secretion of insulin. The IAP activity could likewise be responsible for the attenuation of adrenaline-induced hyperglycaemia observed in pertussis cases by Badr-El-Din et al. (1976). A slight, statistically significant, elevation in plasma insulin, without hypoglycaemia, was detected by Hannik and Cohen (1979) in healthy children given pertussis vaccine - which normally contains a small amount of active PT (Redhead and Seagroatt, 1986).

The object of the present pilot study was to determine whether plasma insulin levels are significantly elevated in hospitalised patients with pertussis and, in turn, whether this is accompanied by hypoglycaemia that might contribute to the complications of the disease. We have, therefore, measured insulin and glucose concentrations in the plasma of pertussis patients and compared them with plasma from children admitted to the same hospital during the same period but suffering from other infectious diseases. We also measured calcium, magnesium and phosphate in the same 
samples, because a previous study (Regan and Tolstoouhov, 1936) suggested that blood constituents other than glucose might be altered in whooping cough.

\section{Patients and Methods}

\section{Study design}

The study (approved by the Glasgow Northern District Ethical Committee) involved 51 children, aged between 1 month and 3 years, admitted to the Infectious Diseases Unit at Ruchill Hospital, Glasgow, between January 1984 and March 1986. Twenty-four children were diagnosed (Walker et al., 1981) as having pertussis by the criterion of a typical paroxysmal cough and at least one of the following: (a) a whoop and a duration of 21 days or more; (b) a whoop and a pronounced lymphocytosis; and (c) isolation of $B$. pertussis from a pernasal swab. The remaining 27 children who served as controls were diagnosed as having an infectious disease other than pertussis. Patients with gastro-enteritis were excluded from the study because of their different dietary management. When a blood sample was taken for medical reasons from each child, either at admission or next morning, an extra 1-2 $\mathrm{ml}$ were taken for insulin and glucose estimation.

Because of ethical considerations we were restricted, with each patient, to a single specimen of blood whose timing could not be controlled. Therefore, we recorded the time interval between sampling and the last feeding. Each blood specimen was dispensed into three different anticoagulants (Sarstedt, 68 Boston Road, Leicester LE4 1AW): lithium-heparin, heparin-fluoride and EDTA (for leukocyte count). The fluoride-containing tubes were used primarily to preserve the glucose concentration where the separation of plasma might be delayed (although, in fact, all plasmas were separated within $2 \mathrm{~h}$ and stored at $-20^{\circ} \mathrm{C}$ ). Because we were uncertain about the best anticoagulant in samples for insulin assay, blood was also collected in lithium-heparin.

\section{Biochemical determinations}

Immunoreactive insulin (IRI) was assayed by the double-antibody method of Hales and Randle (1963) with antibody, ${ }^{125} \mathrm{I}$-insulin and human insulin standard (kit from Amersham, UK) and membrane filters (N25/45/ UP, Oxoid). Glucose was determined enzymically with a Beckman Glu-2 Glucose Analyser; calcium and magnesium were determined by atomic absorption measurements in a Perkin-Elmer 3030 spectrophotometer, and phosphate was assayed by the method of Chen et al. (1956).

\section{Statistical analysis}

Plasma IRI values are approximately log-normally distributed and were, therefore, summarised as the geometric means with $95 \%$ confidence limits (Furman et al., 1981). Plasma glucose values, which are approximately normally-distributed, were summarised as the arithmetic mean and $95 \%$ confidence limits. Statistical analysis of group-means by Student's $t$ test was done on the logarithms of the IRI values and on the glucose data without transformation. Analysis of the calcium, magnesium and phosphate results was done as for glucose.

\section{Results}

\section{Clinical observations}

The 24 pertussis cases $(12 \mathrm{~m}, 12 \mathrm{f})$ did not differ significantly in sex distribution from the controls $(16 \mathrm{~m}, 11 \mathrm{f})$ and showed a similar median age ( 7 and 9 months respectively) and range of ages (1-36 and 2-30 months, respectively). The times of blood sampling in relation to previous feeding were also similar in median (115 and 119 min respectively) and in range (15-315 and 10-450 $\mathrm{min}$ respectively). Of the 24 pertussis cases, $15(62 \%)$ yielded $B$. pertussis from pernasal swabs, 10 had lymphocytosis as previously defined (Walker et al., 1981), and 15 had a duration of at least 21 days. The two groups differed, however, in their duration of stay in hospital, the pertussis cases having a median of 11 days (range 0-24) and the controls a median of 6 days (range 2-13). Among the pertussis cases, the complications or superimposed illnesses were: cyanosis (7), convulsions (2), pneumonia (5), adenovirus infection (2), candidiasis (1), diarrhoea (4) and otitis media (1). None of the pertussis cases had been vaccinated.

Of the 27 non-pertussis controls, the diagnoses were : upper respiratory tract infection (8), bronchitis and bronchiolitis (8), rotavirus infection (2), tonsillitis and otitis media (3), bronchopneumonia (1), candidiasis (1), scabies (1), "viral illness" (1), unexplained pyrexia (1) and mitral incompetence (1). Of the controls, 11 had been vaccinated against pertussis, 14 had not, and the vaccination status of 2 was unknown. The time between vaccination and admission to hospital was not known.

\section{Insulin and glucose levels in plasma}

The table presents the results of the analyses of plasma IRI and glucose in the specimens obtained in the two anticoagulants, heparin-fluoride and lithium-heparin. With both anticoagulants, the 
mean IRI level of the plasma samples from pertussis patients was higher than that of the control plasmas, but only with the samples anticoagulated with heparin-fluoride was this difference statistically significant $(p=0 \cdot 017)$. The elevation of the pertussis mean IRI over the control mean IRI was $49 \%$ with the heparin-fluoride samples and $36 \%$ with the lithium-heparin samples, an average elevation of $43 \%$.

For comparing the two anticoagulants, results from both groups of patients were analysed by a paired-sample $t$ test. The overall average IRI of the lithium-heparin samples was $24 \%$ higher than that of the heparin-fluoride samples, a difference which was highly significant $(\mathrm{p} \ll 0 \cdot 01)$.

The significantly elevated mean IRI level in the samples from pertussis patients was not accompanied by hypoglycaemia. Indeed, as shown in the table, the mean glucose levels in these samples was $7 \%$ and $5 \%$ higher (in the fluoride and lithium samples respectively) than in the controls-a difference that was not statistically significant. Paired-sample $t$ test of the glucose levels in all the heparin-fluoride samples, compared with the lithium-heparin samples, showed that, unlike the IRI results, there was no significant difference due to the type of anticoagulant.

The mean levels of plasma calcium, magnesium and phosphate did not differ significantly between the pertussis and control groups. Respectively the means (in mmol/1) and $95 \%$ confidence limits for pertussis and control were, for calcium : $2 \cdot 30(2 \cdot 17$, $2 \cdot 43)$ and $2.31(2 \cdot 11,2.50)$; magnesium: $0.83(0.79$, $0.88)$ and $0.87(0.64,1.09)$; and phosphate: 0.042 $(0.035,0.049)$ and $0.036(0.032,0.04)$.

\section{Discussion}

This study suggests that plasma insulin levels may be slightly elevated in pertussis, but that hypoglycaemia, if it occurs at all, is uncommon, even in cases admitted to hospital. Ideally, completely normal infants should have been used as controls, but this was precluded by ethical considerations, as was the obtaining of a series of samples at defined times after feeding and after paroxysmal coughing. Apart from their specific illness, both groups had similar age and sex distribution, and similar food, and they had their blood samples taken over a similar spread of times since feeding. Thus we can tentatively attribute the slightly elevated mean plasma-insulin to pertussis. Recent pertussis-vaccination of some of the controls may have biased the study against demonstrating a more marked difference between the two groups. How-
Table. Plasma levels of insulin and glucose in pertussis and control patients; and influence of different anticoagulants

\begin{tabular}{|c|c|c|c|}
\hline \multirow[b]{3}{*}{ Patients } & \multicolumn{3}{|c|}{ Mean* plasma level $(95 \%$ confidence limits $)$ of } \\
\hline & $\begin{array}{l}\text { immuno-reactive insulin } \\
{[\mu \mathrm{U} / \mathrm{ml}] \text { in samples with }}\end{array}$ & \multicolumn{2}{|c|}{$\begin{array}{l}\text { glucose [mmol] } \\
\text { in samples with }\end{array}$} \\
\hline & $\begin{array}{ll}\text { heparin- } & \text { lithium- } \\
\text { fluoride } & \text { heparin }\end{array}$ & $\begin{array}{l}\text { heparin- } \\
\text { fluoride }\end{array}$ & $\begin{array}{l}\text { lithium- } \\
\text { heparin }\end{array}$ \\
\hline $\begin{array}{c}\text { Pertussis } \\
n=24 \\
\text { Control } \\
n=27\end{array}$ & $\begin{array}{cc}13 \cdot 3 & 15 \cdot 5 \\
(10 \cdot 2-17 \cdot 3) & (11 \cdot 8-20 \cdot 5) \\
8 \cdot 9 & 11 \cdot 4 \dagger \\
(7 \cdot 2-11 \cdot 0) & (8 \cdot 6-15 \cdot 2) \\
\text { Statistical significance } \\
\text { between } \\
0.017 & 0.12\end{array}$ & $\begin{array}{c}5 \cdot 19 \\
(4 \cdot 80-5 ; 6) \\
4 \cdot 85 \\
(4 \cdot 50-5 \cdot 20) \\
e(p) \text { of the } \\
\text { ind control p } \\
0 \cdot 20\end{array}$ & $\begin{array}{c}5 \cdot 00 \\
(4 \cdot 58-5 \cdot 41) \\
4 \cdot 78 \ddagger \\
(4 \cdot 33-5 \cdot 24) \\
\text { ifference } \\
\text { atients } \\
\quad 0.48\end{array}$ \\
\hline
\end{tabular}

* Geometric mean for insulin, arithmetic mean for glucose (see text).

† Only 21 specimens.

$\ddagger$ Only 22 specimens.

ever, there were no significant differences, for any measured value, between vaccinated and nonvaccinated controls. An unresolved issue is the difference in measured insulin concentrations between the plasma samples collected in the two heparin-containing anticoagulants.

The modest hyperinsulinaemia seen in the pertussis patients was associated with a slight (nonsignificant) increase, rather than a decrease, in plasma glucose. This is in contrast to the results of our studies in B. pertussis-infected mice which have shown consistently both hyperinsulinaemia and hypoglycaemia (Pittman et al., 1980; Furman et al., 1981, 1986). We do not know if the hyperinsulinaemia in patients with pertussis is mediated via the same mechanisms as those in mice. Hyperinsulinaemia in the mouse follows two patterns: an exaggerated plasma insulin response to feeding, and a qualitatively abnormal plasma insulin response to stress (Furman et al., 1981, 1986; Sidey et al., 1987). The latter results in a very marked, but transient, hyperinsulinaemia following exposure to stresses such as anoxia or ether. The possibility remains that in a small proportion of pertussis patients there may be a marked, but transient, release of insulin in response to either food or a coughing paroxysm but such hyper-reactive patients were not observed in this small series.

Our results differ from those of Regan and Tolstoouhov (1936) who reported hypoglycaemia, and also lower plasma phosphate, in children with whooping cough. However, these authors measured 
the whole blood-sugar by a method that estimates total reducing sugar, rather than glucose specifically. A more important factor may be the much older children studied by Regan and Tolstoouhov (78\% more than 36 months old) compared with those in our study (median age 7 months). Indeed, we have found that metabolic responses to PT are developed fully only in adult mice (Sidey, Wardlaw and Furman, unpublished observations). In agreement with the present study, Badr-El-Din et al. (1976) found no relative hypoglycaemia in infants with pertussis. However, these authors reported a clearly impaired hyperglycaemic response to adrenaline, a condition seen also in the $B$. pertussisinfected mouse (Furman et al., 1981).

The observations reported here suggest that PT released by $B$. pertussis during its growth in the human respiratory tract, in addition to causing lymphocytosis, may affect also the insulin-secreting cells in the pancreas. A notable feature of pertussis as a disease is the wider range in severity of

\section{REFERENCES}

Badr-El-Din M K et al. 1976 The beta-adrenergic receptors in pertussis. Journal of Tropical Medicine and Hygiene 79:213217.

Chen P S, Toribara T Y, Warner H 1956 Microdetermination of phosphorus. Analytical Chemistry 28: 1756-1758.

Furman B L, Sidey F M, Wardlaw A C 1986 Role of insulin in the hypoglycaemic effect of sublethal Bordetella pertussis infection in mice. British Journal of Experimental Pathology 67: 305-312.

Furman B L, Wardlaw A C, Stevenson L Q 1981 Bordetella pertussis-induced hyperinsulinaemia without marked hypoglycaemia: a paradox explained. British Journal of Experimental Pathology 62:504-511.

Hales C N, Randle P J 1963 Immunoassay of insulin with insulin-antibody precipitate. Biochemical Journal 88:137146.

Hannik C A, Cohen H 1979 Changes in plasma insulin concentration and temperature of infants after pertussis vaccination. pp 297-299 In: Manclark C R, Hill J C (eds) International symposium on pertussis. US DHEW Publication no (NIH) 79-1830, Washington D.C.

Pieroni R E, Stevens D L, Stojanovic A, Levine L 1971 Investigation of cutaneous histamine sensitivity in children with pertussis. International Archives of Allergy and Applied Immunology 41 : 940-944.

Pittman M 1979 Pertussis toxin: the cause of the harmful effects symptoms in different patients - from a mild cough to severe and life-threatening paroxysmal coughing, and from a normal white-cell pattern to a massive lymphocytosis. It is possible, therefore, that a similar range of responsiveness occurs at the pancreas and that some--perhaps only a fewchildren may have a much more pronounced hyperinsulinaemia than seen in our small series. Such an occurrence could conceivably cause hypoglycaemia and thus contribute to the neurological sequelae of whooping cough in some cases.

We are grateful to the Scottish Home and Health Department for financial support; to junior medical staff at Ruchill Hospital for collecting the samples; to Dr R. J. Fallon and to Mr I. Marshall, Department of Laboratory Medicine, Ruchill Hospital, for arranging to separate the plasma samples; and to Drs W. C. Love, D. H. Kennedy and I. W. Pinkerton for access to patients under their care. We also thank Mrs Barbara Ottaway of Strathclyde University, and Dr D. Halls of Glasgow Royal Infirmary, for assistance with the calcium and magnesium determinations. This study owes much to Dr Margaret Pittman, whose encouragement and enthusiasm were invaluable.

and prolonged immunity of whooping cough. A hypothesis. Reviews of Infectious Diseases 1:401-412.

Pittman M 1984 The concept of pertussis as a toxin-mediated disease. Pediatric Infectious Diseases 3:467-486.

Pittman M, Furman B L, Wardlaw A C 1980 Bordetella pertussis respiratory tract infection in the mouse: pathophysiological responses. Journal of Infectious Diseases 142:56-66.

Redhead K, Seagroatt V 1986 The effects of purified components of Bordetella pertussis in the weight gain test for the toxicity testing of pertussis vaccines. Journal of Biological Standardization 14:57-65.

Regan J C, Tolstoouhov A 1936 Relations of acid-base equilibrium to pathogenesis and treatment of whooping cough. New York State Journal of Medicine 36:1075-1087.

Sanyal R K 1960 Histamine sensitivity in children after pertussis infection. Nature 185 : $537-538$.

Sidey F M, Wardlaw A C, Furman B L 1987 Hypoglycaemia and acute stress-induced hyperinsulinaemia in mice infected with Bordetella pertussis or treated with pertussis toxin. Journal of Endocrinology 112: 113-122.

Ui M, Nogimori K, Tamura M 1985 Islet-activating protein, pertussis toxin: subunit structure and mechanism for its multiple biological actions. In: Sekura R D et al. (eds) Pertussis toxin. Academic Press, Inc. New York pp 19-44.

Walker E, Pinkerton I W, Love W C, Chaudhuri A K R, Datta J B 1981 Whooping cough in Glasgow 1969-1980. Journal of Infection 3:150-158.

Wardlaw A C, Parton R. 1982 Bordetella pertussis toxins. Pharmacology and Therapeutics 19:1-53. 